Résumés des conférences et travaux

\title{
Aux frontières orientales de Byzance
}

Textes, manuscrits, types d'écriture grecque

Francesco D'Aiuto

\section{OpenEdition \\ Journals}

Édition électronique

URL : https://journals.openedition.org/ashp/2910

DOI : 10.4000/ashp.2910

ISSN : 1969-6310

Éditeur

Publications de l'École Pratique des Hautes Études

Édition imprimée

Date de publication : 1 septembre 2019

Pagination : 75-87

ISSN : 0766-0677

Référence électronique

Francesco D'Aiuto, «Aux frontières orientales de Byzance », Annuaire de l'École pratique des hautes études (EPHE), Section des sciences historiques et philologiques [En ligne], 150 | 2019, mis en ligne le 07 juin 2019, consulté le 06 juillet 2021. URL : http://journals.openedition.org/ashp/2910 ; DOI : https:// doi.org/10.4000/ashp.2910 


\title{
AUX FRONTIÈRES ORIENTALES DE BYZANCE
}

\author{
TEXTES, MANUSCRITS, TYPES D'ÉCRITURE GRECQUE \\ Conférences de M. Francesco D'Aiuto, \\ université de Rome « Tor Vergata», \\ directeur d'études invité
}

\section{Introduction}

Ce cycle de conférences se propose d'explorer, à la lumière aussi de quelques recherches nouvelles en cours, un territoire des études consacrées au Moyen Âge grec qui ne paraît pas avoir encore été analysé comme il mériterait de l'être : celui des textes, des manuscrits et des types d'écriture des régions orientales byzantines (ou ex-byzantines), considérés sous le double point de vue des formes typiques d'une expression locale et des attestations de contacts multiples avec les différentes cultures de l'Orient chrétien. En effet, exception faite pour quelques études remarquables, surtout codicologiques, effectuées dans une perspective comparatiste entre le monde byzantin et les divers domaines orientaux ${ }^{1}$, la portée culturelle réelle des phénomènes de bilinguisme (ou, parfois, de multilinguisme) et, surtout, de digraphisme à Byzance paraît encore largement sous-estimée : dans les études conduites classiquement sur la littérature ou la paléographie byzantine, les intersections relevées entre d'une part la civilisation et la culture graphique grecques médiévales et d'autre part les autres cultures de l'Orient méditerranéen sortent rarement d'une approche générale et floue pour devenir ellesmêmes les objets de la recherche. Ces conférences se proposent de montrer comment - sans être des orientalistes nous-même, mais en nous efforçant de regarder avec attention ce type de témoignages et, surtout, en recourant à l'expérience et à la collaboration des collègues orientalistes - on peut jeter un regard pertinent sur l'incidence que cette réalité multiethnique et multiculturelle complexe eut sur la culture écrite qui, pendant de nombreux siècles, fut incarnée par l'Empire byzantin et, après sa fin, par son rayonnement culturel et spirituel persistant dans la Méditerranée orientale.

Que Byzance fut une réalité plurielle - pour le dire ainsi -, profondément marquée par des phénomènes de coexistence entre peuples et cultures différents, est une notion qui est répétée au début de tous les traités d'histoire byzantine. En effet, on pourrait dire que Byzance a représenté un modèle de réalité étatique universaliste du point de vue de l'idéologie, et supranationale sous l'aspect du peuplement, fondée comme elle était sur une société identitairement composite. Pendant tout le Moyen Âge - au cours des siècles pendant lesquels l'Europe occidentale se faisait le laboratoire de création des différentes identités nationales qui sont à la base de ses réalités étatiques d'aujourd'hui, et pendant lesquels on élaborait la notion même d'État-nation, où l'identité de chaque peuple est marquée par une même langue, une même foi, et des frontières territoriales bien délimitées -, l'Empire byzantin représenta une

1. Voir p. ex. Recherches de codicologie comparée. La composition du codex au Moyen Âge, en Orient et en Occident, éd. P. Hoffmann, C. Hunzinger, Paris, 1998. 
expérience extraordinaire de cohabitation et d'interaction - pas toujours pacifique, bien sûr - entre de nombreuses ethnies caractérisées par des traditions, des cultures et des langues différentes.

On pourrait même parler d'une sorte de « double appartenance », du point de vue identitaire, des sujets de l'Empire byzantin. Par exemple, un Arménien qui vivait dans les territoires byzantins se sentait, certes, arménien du point de vue de la culture et de l'appartenance religieuse - soit qu'il fût un arménien orthodoxe lié à son Église nationale non-chalcédonienne, soit qu'il fût un chalcédonien en communion avec l'Église de Constantinople -, mais il devait naturellement se sentir en même temps comme faisant partie de l'Empire, même si ce sentiment d'appartenance a pu grandement varier selon l'époque et la région. Mais, à vrai dire, on pourrait même aller plus loin, et dire que c'est la question même d'une appartenance ethnique en termes identitaires qui est peut-être mal posée, si on essaie de l'appliquer à l'Orient chrétien pendant le Moyen Âge. En effet, la notion même d'identité nationale peut difficilement être utilisée quand il est question d'une époque et de régions auxquelles cette mentalité était substantiellement étrangère.

Si cette structure multiethnique est bien un caractère fondamental de l'Empire byzantin, une considération attentive de celle-ci devrait figurer dans les présupposés indispensables à toute étude de son héritage littéraire, artistique, scientifique et de sa culture matérielle. Cette affirmation est, certes, largement partagée dans les études modernes sur le monde byzantin, du moins en théorie. Cependant, on peut déplorer que les implications concrètes de ce postulat, sur le plan des phénomènes historiques et culturels, ne soient pas toujours gardées à l'esprit dans la pratique quotidienne de la recherche historique, littéraire, et paléographique aussi. Nous, érudits modernes, sommes habitués, en Occident, à nous confronter surtout avec la culture gréco-latine classique, tandis que, généralement, nous ne sommes pas bien introduits aux civilisations de l'Orient méditerranéen qui, avec celle-ci, constituaient l'arrière-plan et, en même temps, l'âme de Byzance : en effet, nous ne sommes pas suffisamment entraînés à nous apercevoir des phénomènes d'échange ou de partage entre la culture byzantine et les autres cultures de l'Orient chrétien, et, sur un plan plus général, nous ne sommes pas toujours capables de déceler et de mettre en valeur des convergences ou des consonances qui sont parfois très significatives.

\section{Byzance et la région sub-caucasienne : interactions textuelles, graphiques, codicologiques et ornementales}

Grâce à une série d'exemples significatifs, déjà signalés ou bien inédits, on peut essayer de dresser un panorama des interactions linguistiques, graphiques, codicologiques et artistiques entre la production livresque grecque du Moyen Âge et les productions arménienne et géorgienne. Des recherches nouvelles démontrent en effet qu'une vaste circulation de livres et d'habitudes graphiques, de techniques de confection matérielle et de choix décoratifs a constamment existé entre Byzance et la région caucasienne, en raison du rôle de point de repère central que la civilisation byzantine exerça pendant tout le Moyen Âge par rapport aux autres cultures sœurs de l'Orient chrétien. Ce ne furent cependant pas des phénomènes de rayonnement à sens unique, 
à savoir allant exclusivement du "centre » aux «périphéries »: les contacts et les échanges ont été dans une certaine mesure multidirectionnels car ils furent la conséquence naturelle de la cohabitation profondément ancrée d'ethnies et de cultures différentes dans l'Empire byzantin et même en son cœur, voire dans sa capitale. Ces interactions furent alimentées et favorisées par la présence d'une composante tant arménienne que géorgienne forte et importante dans le système des familles aristocratiques de l'Empire et, par conséquent, dans les hautes hiérarchies civiles et militaires et, en général, dans l'intelligentsia byzantine : formés aux études supérieures grecques, mais naturellement bilingues et dépositaires aussi d'un héritage culturel et littéraire alloglotte également raffiné, les membres de ces élites furent les principaux médiateurs d'un dialogue culturel entre ethnies qui fut fécond et ininterrompu, et dont on peut observer des traces bien visibles dans les textes et les manuscrits grecs, arméniens et géorgiens.

Pour essayer de clarifier ce point, on peut partir d'un exemple tiré de la littérature byzantine : les épîtres de Léon, évêque de Synada, en Asie Mineure, qui vécut à la fin $\mathrm{du} \mathrm{X}^{\mathrm{e}}$ siècle et qui est l'un des épistolographes les plus célèbres et les plus plaisants du Moyen Âge byzantin. Ses lettres ont été publiées pour la première fois par le père Jean Darrouzès en 1960, dans un imposant travail dédié aux Épistoliers byzantins $d u$ $X^{e}$ siècle $^{2}$. Or une des réactions les plus inattendues au lendemain de la parution du livre de Darrouzès fut la publication d'un long article par Louis Robert : le spécialiste renommé d'épigraphie grecque classique consacra pas moins de 140 pages à éclairer la signification de seulement trois mots rares et obscurs dans tant de lettres de Léon de Synada $^{3}$. Par un véritable tour de force d'érudition, Robert entreprenait la recherche de données historiques cachées dans la prose de l'épistolier byzantin, qu'il essayait d'interpréter surtout à la lumière de ses connaissances de l'Antiquité classique, sans tenir compte, cependant, de la réalité multiethnique de la région anatolienne où Léon de Synada avait vécu et écrit ses lettres.

Pour se limiter à un seul exemple, il est possible de se référer à la lettre 27 de Léon de Synada, adressée à Grégoire métropolite de Nicée : dans ce texte, Louis Robert se concentre sur une phrase qui attire l'attention par un curieux serment. Léon écrit qu'il n'a pas pu bien comprendre ce que son correspondant lui avait écrit dans un style trop élevé, et puis il déclare :

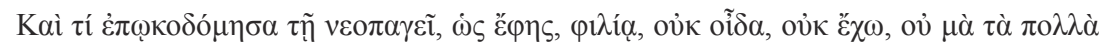

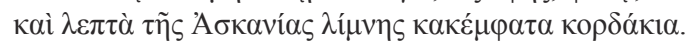

Et qu'est-ce que j'ai ajouté au nouvel édifice de notre amitié, comme tu l'as défini, je ne le sais pas, je ne peux pas le comprendre : je le jure par les innombrables petits kordakia, qui sont kakemphata, du lac Ascanien!

2. Épistoliers byzantins du Xe siècle, éd. J. Darrouzès, Paris, 1960 (Archives de l'Orient chrétien 6). Les épîtres de Léon ont étés rééditées plus récemment par M. Pollard Vinson, The Correspondence of Leo, Metropolitan of Synada and Syncellus, Washington (D.C.), 1985 (Corpus Fontium Historiae Byzantinae 23; Dumbarton Oaks Texts 8).

3. L. Robert, «Les kordakia de Nicée, le combustible de Synnada et les poissons-scies. Sur des lettres d'un métropolite de Phrygie au $\mathrm{x}^{\mathrm{e}}$ siècle. Philologie et réalités », Journal des Savants, juillet-décembre 1961, p. 97-166, et janvier-juin 1962, p. 5-74, réimpr. dans L. Robert, Opera minora selecta. Épigraphie et antiquités grecques, VII, Amsterdam, 1990, p. 1-140. 
L'expression est fort originale, et le mot корбóкı est absent de tous les lexiques du grec ancien et médiéval. C'est évidemment une plaisanterie, avec une allusion humoristique à la cité dont le correspondant de Léon était l'évêque métropolite, le lac Ascanien étant le lac qui baignait la cité de Nicée, à savoir l'actuelle Iznik. Cette plaisanterie est renforcée par la reprise comique, avec une variation étudiée, d'un type de serment qui était typique du grec attique, et qui est très répandu dans les textes classiques. Un Grec de l'Antiquité pouvait jurer, comme Louis Robert le dit bien,

« par l'oie, le chien, le bélier, etc. » (...) «par le platane », (...) « par le chou », etc. (...). Ce genre de serment est susceptible de variations infinies, c'est affaire d'ingéniosité. Il est ici renouvelé par la mention d'une faune remarquable du pays du correspondant auquel s'adresse Léon de Synnada.

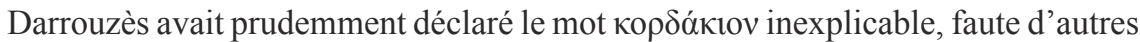
occurrences dans la littérature grecque classique et byzantine. Louis Robert, pour sa part, essaya de trouver le sens du terme, en faisant un long détour par la littérature classique et l'érudition antiquaire : avant tout, il suppose que ces kordakia sont des animaux, et plus spécifiquement des poissons; mais l'un des adjectifs liés à ce mot dans la lettre de Léon de Synada est $\kappa \alpha \kappa \varepsilon ́ \mu \varphi \alpha \tau o \zeta$, adjectif composé, qui se retrouve dans le grec classique aussi, et qui a bien la valeur de « de mauvaise réputation », ou encore de "vulgaire», comme le dit Robert (mais qui signifie d'abord " cacophonique », ou bien " désagréablement bruyant» : et si l'adjectif a bien son sens de base ici, on ne voit pas comment on pourrait l'appliquer à un poisson!). Le savant helléniste, en effet, a été immédiatement frappé par la proximité du mot kordakion de notre épistolier byzantin avec le mot grec classique kordax - la célèbre danse licencieuse de l'Antiquité -, proximité sur laquelle il a basé son interprétation sans trop réfléchir aux possibles alternatives : de là vient aussi sa traduction de kakemphatos, dans ce contexte, par « licencieux », ou bien «vulgaire» (signification dont on ne voit pas bien comment elle pourrait s'appliquer à l'apparence ou aux mouvements d'un poisson, aussi frénétiques qu'ils puissent être!). Après s'être donc informé minutieusement sur les sortes de poissons du lac de Nicée d'après les sources classiques, Robert en conclut que le candidat idéal pour y reconnaitre le kordakion serait un petit poisson, l'ablette, qui fait de petits sauts soudains en sortant de l'eau quand elle chasse les mouches.

Cette démonstration appelle toutefois plusieurs objections et, en premier lieu, une remarque banale : les lacs ne sont pas peuplés seulement de poissons! Et surtout, en nous plaçant sur un plan plus élevé, l'héritage grec classique n'est pas la seule composante qui anime et vivifie la culture et la vie matérielle de Byzance : on ne devrait jamais oublier le tréfonds oriental de la civilisation byzantine. Dans le cas qui nous intéresse, on ne doit pas négliger le fait que, dans la région de l'Anatolie dans laquelle aussi bien Synada que Nicée étaient situées, la population comportait une très importante composante arménienne. On ne devrait alors pas trop s'étonner qu'une solution alternative pour l'énigme des kordakia puisse jaillir de la simple consultation d'un dictionnaire d'arménien classique. En effet, on trouve le mot arménien gort, dont le pluriel est gortk' (qui doit être prononcé kordk' selon la prononciation occidentale de l'arménien, qui tendait à s'imposer déjà aux $\mathrm{IX}^{\mathrm{e}}$ et $\mathrm{X}^{\mathrm{e}}$ siècles dans l'Ouest 
de l'Anatolie, à savoir dans la région où tant Nicée que Synada se trouvaient) : ce mot signifie « grenouilles », ce qui s'accorde très bien avec le contexte naturel du lac Ascanien qui est évoqué par la lettre de Léon de Synada. En reprenant le texte, on pourra donc l'interpréter comme ceci :

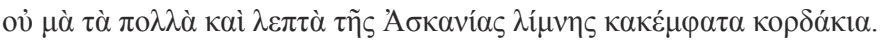

je le jure par les innombrables petites grenouilles, désagréablement bruyantes, du lac Ascanien!

C'est une allusion ironique à l'ennuyeux coassement des milliers de petites grenouilles du lac de Nicée, qui devait probablement fatiguer les oreilles de ceux qui habitaient dans les alentours. Mais, dans la lettre de Léon de Synada, l'adjectif kakemphatos n'indique pas seulement le bruit lancinant fait par l'animal, il a aussi, en même temps, une autre fonction. Il sert à caractériser comme étranger le mot kordk', qui, en tant que dérivé de l'arménien, a quelque chose de barbare pour des oreilles grecques «bien éduquées ».

Du reste, comme nous allons le montrer dans une étude plus détaillée qui est en préparation, l'exemple que nous venons d'analyser n'est pas le seul cas d'emploi, par Léon de Synada ou par d'autres écrivains méso-byzantins, de mots arméniens translittérés dans leur prose grecque. Faut-il alors penser que ces intellectuels connaissaient l'arménien? Nous n'avons pas de notices biographiques précises sur l'origine familiale de nombre de ces écrivains mais il n'y aurait pas lieu de s'étonner qu'ils aient pu parfois être des membres de familles arméniennes intégrées et au service de l'Empire byzantin. En tout cas, Léon de Synada devait avoir une connaissance au moins passive de l'arménien, ce qui vaut aussi pour ses correspondants : en effet, Léon n'a pas ressenti le besoin d'expliquer à Grégoire, le métropolite de Nicée, ce qu'étaient les kordk', évidemment parce qu'il savait que Grégoire pouvait bien comprendre le mot arménien, et la plaisanterie qu'il impliquait.

Mais, bien plus que dans les traces linguistiques alloglottes qu'on pourrait mettre en valeur dans la littérature grecque médiévale, ce sont les manuscrits - et la considération de certains aspects paléographiques et codicologiques de la production de livres, soit en grec soit en d'autres langues de l'Orient chrétien - qui nous révèlent la richesse des échanges entre les différentes cultures du «Commonwealth byzantin ». Plus spécifiquement, que du point de vue graphique également les relations entre Byzance et le Caucase aient toujours été très étroites, est une réalité évidente pour peu que l'on considère les modalités de la création même, au début $\mathrm{du} \mathrm{V}^{\mathrm{e}}$ siècle après Jésus-Christ, de l'alphabet arménien, qui est attribuée par les sources historiques arméniennes au saint moine Mesrop-Maštoc'. Au delà des clichés hagiographiques, en lisant entre les lignes mêmes des sources hagiographiques et historiques - telle la Vie de saint Mesrop écrite par son disciple Korioun ou l'Histoire des Arméniens de Moïse de Khorène ${ }^{4}$-, l'alphabet arménien se révèle être une claire adaptation de l'alphabet grec. Ces sources nous relatent de fait qu'à Samosate, où Mesrop s'était

4. J.-P. Mahé (éd.), L'alphabet arménien dans l'histoire et dans la mémoire. Vie de Machtots par Korioun. Panégyrique des Saints Traducteurs par Vardan Areveltsi, Paris, 2018 (Bibliothèque de l'Orient chrétien, 5); A. Mahé, J.-P. Mahé (éd.), Histoire de l'Arménie par Moïse de Khorène, nouvelle traduction de l'arménien classique (d'après V. Langlois), Paris, 1993. 
rendu à la recherche d'une solution pour l'alphabet arménien, un certain calligraphe nommé Rufin - ou Hrop 'anos dans le texte arménien - aida Mesrop en donnant leur forme calligraphique définitive aux lettres de l'alphabet que le saint avait contemplées dans sa vision miraculeuse d'une main qui traçait la série des formes alphabétiques. Du reste, la dérivation de l'alphabet arménien à partir du grec - qui a récemment été contestée par plusieurs savants et qui, d'autre part, n'a jamais été démontrée de façon analytique, comme je souhaite le faire dans le cadre d'un prochain travail d'intérêt paléographique - paraît évidente du point de vue de la morphologie des signes, comme une simple liste de quelques signes alphabétiques de forme presque identique dans les deux langues suffira à le montrer $(A, B \ldots E, Z \ldots \Theta, I \ldots=U, f \ldots G, 2 \ldots \rho$, $\mathrm{r} . .$.$) .$

En tout cas, si la création même du système graphique relie étroitement au monde gréco-byzantin, dès leur origine, l'alphabet arménien et, selon les sources arméniennes, l'alphabet géorgien également, on peut observer de plus que ce lien génétique ne cessera jamais de produire des interactions non seulement sur le plan graphique, mais aussi sous l'angle des caractéristiques matérielles et artistiques de la production de manuscrits. Parmi les nombreux exemples qu'on pourrait évoquer à ce propos, on peut citer en premier lieu le cas de la dérivation du signe d'interponction arménien nommé but ' (') à partir du signe grec équivalent en forme de clou qu'on retrouve dans les manuscrits byzantins $\left(^{\wedge}\right)$ et qui a reçu le nom de « chiodo Follieri » en l'honneur de la savante italienne Enrica Follieri, qui en a signalé l'existence et expliqué l'emploi en $1971^{5}$. Les deux signes suivent les mêmes règles d'usage dans les deux langues mais ont eu des destins différents : le clou a été abandonné assez tôt en grec, tout en résistant un peu plus longuement dans l'Italie méridionale de l'époque méso-byzantine, alors qu'en arménien le but 'est utilisé encore aujourd'hui, même dans les textes imprimés, pour exprimer toujours la même fonction de pause moyenne, souvent de valeur suspensive ${ }^{6}$.

Sur un plan différent, mais complémentaire, il vaut la peine, enfin, de signaler aussi les échanges de motifs ornementaux ou iconographiques qui ont lieu au niveau de l'art de la miniature de manuscrits entre l'Empire byzantin et les mondes arménien et géorgien ${ }^{7}$, ou encore la production mixte, dans un même atelier et par les mêmes mains, de manuscrits enluminés soit grecs soit géorgiens, phénomène dont on a signalé plusieurs cas qui s'échelonnent entre le $\mathrm{XI}^{\mathrm{e}}$ et le $\mathrm{XV}^{\mathrm{e}}-\mathrm{XVI}^{\mathrm{e}}$ siècle, et qu'on peut

5. E. Follieri, «Un segno di interpunzione in lezionari italo-greci », dans $X I V^{e}$ congrès international des études byzantines, Bucarest 6-12 Septembre 1971. Résumés - Communications, Bucarest, 1971, p. 176.

6. Pour plus de détails, F. D'Aiuto, «"Chiodo", "point and hook", "but"”: metamorfosi di un segno interpuntivo fra Bisanzio, l'Italia e il Caucaso ", dans Per Gabriella. Studi in ricordo di Gabriella Braga, vol. II, éd. M. Palma, C. Vismara, Cassino, 2013, p. 575-599.

7. Voir p. ex. Helmut Buschhausen, Heide Buschhausen, «Kopien des Vani-Evangeliars », Cahiers archéologiques. Fin de l'Antiquité et Moyen Âge 39 (1991), p. 133-152; F. D’Aiuto, «Il libro dei Vangeli fra Bisanzio e l'Oriente: riflessioni per l'età mediobizantina », dans Forme e modelli della tradizione manoscritta della Bibbia, éd. P. Cherubini, Cité du Vatican, 2005 (Littera antiqua 13), p. 309345 et pl. 25-28 h.t.; F. D'Aiuto, «Per un approccio multiculturale alla produzione libraria miniata: Bisanzio e altri "Orienti cristiani”" ", dans Medioevo: la Chiesa e il Palazzo. Atti del Convegno internazionale di studi, Parma, 20-24 settembre 2005, éd. A. C. Quintavalle, Milan, 2007, p. 228-237. 
situer dans la région syro-palestinienne, entre Antioche et Jérusalem ${ }^{8}$ : une région qui a toujours connu une intense activité de production de textes littéraires et de manuscrits dans les dizaines de foyers monastiques géorgiens qui y sont attestés 9 .

\section{Une hymnographie d'allure régionale : Palestine et Syrie, entre manuscrits et textes inédits}

Genre littéraire assez peu cultivé par la recherche en général, l'hymnographie byzantine mériterait d'être l'objet d'une ample enquête tournée vers l'étude du milieu particulièrement significatif qu'est la région syro-palestinienne au sens large. Il est bien connu que c'est de cette vaste région que furent originaires des hymnographes parmi les plus célèbres et les plus vénérés, tels que Romanos le Mélode, André de Crète, Cosmas de Maïouma et Jean Damascène. Mais on ignore encore presque totalement une quantité d'hymnes qui eurent sans doute seulement une circulation locale, et qui restent encore inédits et négligés dans des manuscrits qui sont souvent conservés dans la région même où ils ont été copiés. Ce sont des hymnes parfois très anciens, qui ont totalement disparu de la tradition liturgique postérieure : on a dû cesser de les transcrire assez tôt et ils n'ont donc pas eu la chance d'être inclus dans les éditions imprimées au $\mathrm{XVI}^{\mathrm{e}}$ siècle des livres liturgiques byzantins, dont le répertoire reflète, grosso modo, une sélection de textes qui se précisa et s'imposa de manière assez graduelle - bien que d'une manière qui ne fut jamais ni univoque ni généralisée - uniquement dans la dernière période byzantine.

Dans de nombreux cas, la composition de ces hymnes anciens peut être attribuée à des hymnographes qui furent actifs en Syrie ou en Palestine ou bien au Sinaï : ces textes contribuent donc à éclairer la culture hellénophone de ces régions et documentent, de plus, quelques cas intéressants de bilinguisme de la part de leurs auteurs. En effet, l'étude des manuscrits hymnographiques originaires de ces régions, et du répertoire de chants souvent inédits qu'ils nous transmettent, peut réserver des surprises remarquables. C'est le cas, par exemple, d'un hymne alphabétique très ancien, appartenant à un genre hymnographique archaïque qui était resté tout à fait inconnu jusqu'à la découverte récente de ce texte, en 2006 : c'est l' "hymne Schøyen » (inc.

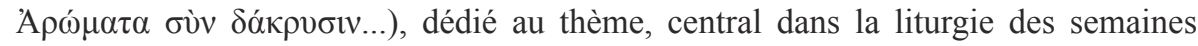

8. A. L. Saminskij, « Gruzinskie i grečeskie rukopisi tret'ej četverti XI v. iz oblasti Antiochii », dans Drevnerusskoe iskusstvo. Iskusstvo rukopisnoj knigi. Vizantija. Drevnjaja Rus', Saint-Peterbourg, 2004, p. 129-148; A. Saminskij, "Georgian and Greek Illuminated Manuscripts from Antioch », dans East and West in the medieval Eastern Mediterranean, I: Antioch from the Byzantine reconquest until the end of the Crusader principality. Acta of the Congress held at Hernen Castle in May 2003, éd. K. N. Ciggaar, Louvain, 2006 (Orientalia Lovaniensia Analecta 147), p. 17-78; F. D’Aiuto, «Libro, scrittura e miniatura fra Bisanzio e il Caucaso: qualche riflessione », dans Scrittura e libro nel mondo greco-bizantino. Atti del corso, Ravello, Villa Rufolo, 6-9 Novembre 2007, éd. C. Casetti Brach, Ravello, 2012, p. 77-108.

9. R. Janin, « Les Géorgiens à Jérusalem », Échos d'Orient 16 (1913), p. 32-38, 211-219; E. Met'reveli, Masalebi Ierusalimis kartuli k'oloniis ist'oriisatvis (XI-XVII ss.) [= Materialy k istorii ierusalimskoj gruzinskoj kolonii (XI-XVII vv.)], Tbilisi, 1962; W. Z. Djobadze, Materials for the Study of Georgian monasteries in the Western Environs of Antioch on the Orontes, Louvain, 1976 (Corpus Scriptorum Christianorum Orientalium, 372; Subsidia, 48). 
suivant Pâques, du récit de la Résurrection, à partir de l'épisode des Femmes pieuses au tombeau du Christ ${ }^{10}$. Cet hymne nous a été conservé, mutilé de sa fin, dans un seul bifolio de parchemin copié en "écriture mixte »- un style d'écriture grecque assez rare et typique des régions du Moyen-Orient vers le $\mathrm{IX}^{\mathrm{e}}$ siècle, comme on le verra plus loin -, un fragment qui appartient aujourd'hui au collectionneur norvégien Martin Schøyen (Oslo-Londres). L' ' hymne Schøyen », probablement composé dans la région palestinienne d'où provient le seul témoin qui le transmet, et peut-être dans la ville sainte de Jérusalem, est le texte-clé - avec quelques autres pièces isolées qui se sont conservées dans les papyrus et les manuscrit médiévaux ou dans quelques versions géorgiennes - d'un genre hymnographique très ancien qui paraît être un précurseur du kontakion, le type d'hymne qui était considéré jusqu'ici comme la première forme de chant polystrophique de l'Église grecque. La création de ce genre d'hymne primitif doit dater au moins du $\mathrm{v}^{\mathrm{e}}$ siècle, tandis que son emploi liturgique ne paraît pas avoir dépassé le début de la période méso-byzantine.

Mais pour illustrer la richesse des nouvelles possibilités qui s'ouvrent à la recherche dans le domaine de l'hymnographie byzantine, on peut aussi mentionner les résultats de premiers sondages effectués sur la figure d'un hymnographe originaire de la Syrie du Nord, qui est resté totalement inconnu et ignoré jusqu'à aujourd'hui, à savoir Abramius le protospathaire d'Antioche. Un récent répertoire de textes hymnographiques inédits signale deux de ses canons dans le manuscrit de Paris, BNF, grec 259 , datable de la première moitié du $\mathrm{XI}^{\mathrm{e}}$ siècle $^{11}$, mais ces hymnes n'ont apparemment pas encore attiré l'attention des savants ( $\mathrm{j}$ 'espère en donner l'édition dans un travail prochain). Le premier hymne, pour la fête des archanges du 8 novembre, est spécifiquement dédié à célébrer le souvenir de l'inauguration de l'église, qui avait été récemment restaurée, de Saint-Michel-l'Archange à Antioche, tandis que l'autre canon d'Abramius, à la date du 27 novembre, est lui aussi dédié à un culte typique de la même ville, à savoir celui de saint Palladius d'Antioche, un ermite et thaumaturge qui a vécu pendant le $\mathrm{IV}^{\mathrm{e}}$ siècle dans une grotte dans le désert près de la ville d'Antioche, et qui était un ami et compagnon d'ascèse de saint Syméon l'Ancien du mont Sinaï. Mais quelles données sur Abramius le protospathaire peuvent être déduites de la lecture de ses deux hymnes? Apparemment on a ici affaire à un dignitaire qui était actif à Antioche après la reconquête byzantine, entre les dernières décennies du $\mathrm{X}^{\mathrm{e}}$ siècle et les premières décennies $\mathrm{du} \mathrm{XI}^{\mathrm{e}}$. Peut-on, alors, mieux déterminer son identité? On retrouve en fait, dans les répertoires prosopographiques du monde arabe chrétien, Ibrāhīm 'l-Anțākī, ou bien Ibrāhīm 'l-Abrotosbatiār ibn Yūḥanna (environ 950-environ 1030), un personnage mentionné dans toutes les histoires littéraires arabo-chrétiennes et qui est connu, avant tout, en tant que traducteur du grec en arabe : il a traduit en arabe un choix des homélies de Grégoire de Nazianze, une partie du $D e$

10. F. D’Aiuto, «Per la storia dei libri liturgico-innografici bizantini: un progetto di catalogazione dei manoscritti più antichi », Bollettino della Badia greca di Grottaferrata, III sér., 3 (2006), p. 53-66. Édition et étude du texte dans F. D’Aiuto, « Un antico inno per la Resurrezione (con nuove testimonianze di "scrittura mista" d'area orientale) », Rivista di studi bizantini e neoellenici, n.s. 45 (2008), p. 3-135, avec 12 pl. h.t.

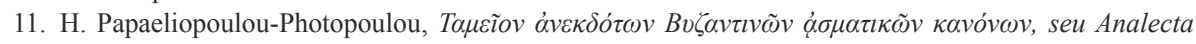
Hymnica Graeca e codicibus eruta Orientis Christiani, I, Athènes, 1996, $\mathrm{n}^{\text {os }} 201$ et 290. 
divinis nominibus du pseudo-Denys l'Aréopagite et quelques sermons de l'Éphrem grec. Mais il est surtout l'auteur d'un texte hagiographique célèbre, qu'il nous dit avoir composé en grec à l'origine et avoir traduit lui-même en arabe : ce texte, dont seule la version arabe nous est parvenue, est la Vie et passion du patriarche melkite d'Antioche (959-966/7) Christophore ${ }^{12}$ : un ouvrage remarquable en tant que source historique, parce qu'il représente un compte rendu - vivant et de première main des dernières années de gouvernement de la dynastie Hamdānide dans la Syrie du Nord, en nous donnant aussi beaucoup d'informations sur la vie politique et religieuse de l'Antioche contemporaine, à la fois avant et immédiatement après la reconquête byzantine. La découverte, maintenant, des deux canons hymnographiques d'Abramius-Ibrāhīm qui sont contenus dans le manuscrit parisien nous permet ainsi d'apprécier la maîtrise de cet auteur également dans l'emploi d'un grec élégant et cultivé, qui peut en fait être comparé avec son arabe, rhétoriquement très élaboré et riche, en même temps, d'échos de la langue grecque. On a donc affaire ici à un cas éclairant d'un bilinguisme parfait qui, à Byzance, devait être souvent la règle, plutôt qu'une exception.

\section{Un type d'écriture grecque à diffusion locale : l' "écriture mixte "}

Identifiée et définie dans ses caractères graphiques seulement dans les années soixante-dix du $\mathrm{Xx}^{\mathrm{e}}$ siècle, l'« écriture mixte » est un curieux amalgame, attesté dès le $\mathrm{VIII}^{\mathrm{e}}-\mathrm{IX}^{\mathrm{e}}$ siècle et jusqu'au $\mathrm{X}^{\mathrm{e}}$ siècle peut-être, qui se situe entre la majuscule grecque de type ogivale inclinée et, pour un nombre de lettres limité et bien délimité, la minuscule. Jusqu'à il y a quelques années, ce type d'écriture particulier était attesté par une poignée de témoins manuscrits qui semblaient l'ancrer solidement dans la région palestino-sinaïtique. Des recherches plus récentes ont enrichi le dossier de cette écriture, dont on connaît maintenant quelques dizaines de témoins qui documentent sa circulation et sa diffusion en Syrie aussi et même, de façon sporadique, dans des milieux orientaux plus éloignés, sur la route de la soie.

L' « écriture mixte » fut identifiée pour la première fois au moment de la fameuse découverte dans le monastère de Sainte-Catherine au Sinaï, en 1975, d'un grand nombre de manuscrits, pour la majeure partie fragmentaires, soit grecs, soit slaves, soit écrits dans presque toutes les différentes langues de l'Orient chrétien : à savoir l'arabe, le géorgien, le syriaque, et même l'albanien du Caucase ${ }^{13}$. Parmi ces manuscrits et fragments, il y en avait dix-sept, plus ou moins étendus, qui témoignaient d'un type d'écriture qui était resté ignoré jusqu'alors : ce sont les quinze manuscrits qui ont reçu, plus tard, les cotes Sin. gr. NE МГ 15, 24, 26, 29, 37, 48, 52, 81, 82, 83, 84, 88, 91, 99 et 109; mais il y en avait également deux où cette écriture était employée seulement comme écriture distinctive ou Auszeichnungsschrift (Sin. gr. NE MГ 2 et 10). Tous ces fragments montraient une écriture qui était fortement apparentée, dans sa structure de base, à la " majuscule ogivale inclinée », dans sa variété typique syro-palestinienne qui est caractérisée par un axe fortement penché vers la droite; toutefois,

12. H. Zayat, « Vie du patriarche melkite d'Antioche Christophore († 967) par le protospathaire Ibrahîm b. Yuhanna. Document inédit du X $\mathrm{x}^{\mathrm{e}}$ siècle », Proche-Orient chrétien 2 (1952), p. 11-38, 333-366.

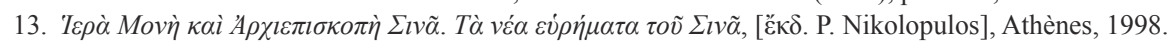


les lettres alpha et $m u$ étaient toujours de forme minuscule, et d'autres lettres aussi, comme kappa en particulier, bien qu'elles fussent majuscules, avaient des ductus assez spéciaux.

Un peu plus tard, en 1983, Lidia Perria a retrouvé cette écriture dans le célèbre manuscrit Vat. gr. 2200, un témoin vénérable, sur papier arabe oriental primitif, de la Doctrina Patrum et d'autres écrits théologiques, copié en "minuscule hagiopolite " tandis que l'" écriture mixte » est utilisée dans le codex seulement comme écriture distinctive en alternance avec la "majuscule ogivale inclinée ${ }^{14}$. Dans des années plus récentes, plusieurs autres témoins de cette écriture ont été signalés, et le dossier compte désormais plus de quarante manuscrits divers, surtout grâce à l'identification de cette écriture dans nombre des fragments découverts au début du $\mathrm{XX}^{\mathrm{e}}$ siècle à Damas dans la Qubbat al-ḩazna, ce qu'on appelle le «Trésor» de la mosquée des Omeyyades ${ }^{15}$.

Les nombreux nouveaux témoins qui ont été repérés nous permettent : a) de mieux reconstruire l'évolution et la chronologie de ce style d'écriture; b) de le localiser non plus seulement au Sinaï - comme, entre autres, le savant grec Panagiotis Nikolopoulos le croyait -, mais dans la région syro-palestinienne tout entière, avec une diffusion remarquable également à Damas et dans la Syrie du Nord; c) de confirmer et préciser la distinction entre les deux variétés du style qui ont été identifiées et classifiées par Lidia Perria en $2002^{16}$, et qu'on pourrait respectivement nommer variété « élégante » (caractérisée par un contraste marqué des modules des lettres, par une infraction assez nette au système bilinéaire et par un ductus plus fin) et variété « compacte » (avec des formes plus trapues mais aussi plus régulières, et un ductus plus lourd).

On peut aussi signaler que, alors que la circulation de cette dernière variété « compacte » de l' " écriture mixte » ne semble attestée, pour l'instant, que dans la région du Sinaï, et qu'elle paraît surtout destinée à une utilisation plus modeste dans de simples livres de chant hymnographique et de littérature ascétique - à savoir dans des livres d'usage typiquement monastique -, la variété «élégante » est plutôt associée à un catalogue de textes plus riche et à des produits de plus haut niveau, qui étaient apparemment destinés, au moins en partie, à l'usage du clergé des grands centres (comme c'est le cas de la Liturgie de Saint Jacques du Vat. gr. 2282), ou à un usage didactique (comme la paraphrase de l'Iliade du Sin. gr. NE МГ 26) ou à la documentation théologique (comme la Doctrina Patrum du Vat. gr. 2200), dans une région de diffusion qui paraît plus vaste, et qui a son centre apparemment déplacé vers le nord, dans la région de Damas ou, peut-être, entre le Sud de la Syrie et la Palestine.

14. L. Perria, «Il Vat. gr. 2200. Note codicologiche e paleografiche », Rivista di studi bizantini e neoellenici, n.s. 20-21 (1983-1984), p. 25-68, avec 6 pl. h.t., réimpr. dans Tra Oriente e Occidente. Scritture e libri greci fra le regioni orientali di Bisanzio e l'Italia, éd. L. Perria, Rome, 2003 (Testi e studi bizantino-neoellenici 14), p. 3-46, avec 6 pl. h.t.

15. F. D'Aiuto, « Un antico inno per la Resurrezione »; et surtout F. D’Aiuto, « La "scrittura mista” maiuscolo-minuscola d'area mediorientale », dans Griechisch-byzantinische Handschriftenforschung. Traditionen, Entwicklungen, neue Wege [= Proceedings of the 8th International Colloquium for Greek Paleography, Hamburg, 22-28 September 2013], éd. C. Brockmann, D. Deckers, D. Harlfinger, S. Valente, Berlin, Boston (en cours d'impression), p. 147-171, 735-739 (pl. 1-5), avec bibliographie.

16. L. Perria, «Libri e scritture tra Oriente bizantino e Italia meridionale », Rivista di studi bizantini e neoellenici, n.s. 39 (2002), p. 157-187: 179. 


\section{IV. À la recherche de Graeca dans les manuscrits orientaux : digraphisme, traces alloglottes, palimpsestes}

Les manuscrits du Caucase - arméniens et géorgiens - et du Moyen-Orient - surtout arabes et syriaques - peuvent réserver de belles trouvailles aux chercheurs qui s'occupent de philologie grecque ou de paléographie byzantine et qui s'intéressent aux interactions de la civilisation de Byzance avec les différentes cultures de l'Orient chrétien. On peut, par exemple, considérer sous cet angle quelques exemples significatifs d'allographie, comme le célèbre papyrus Parisinus arménien 332 : il contient des exercices simples de langue grecque qui, dans le $\mathrm{VI}^{\mathrm{e}}$ siècle ou au début du VII ${ }^{\mathrm{e}}$, ont été transcrits en caractères arméniens par un Arménien stationné en Égypte (un marchand peut-être, ou un soldat), qui ne devait pas encore être complètement familier de l'alphabet grec. Un tel document est d'un intérêt extrême tant du point de vue linguistique que sous l'aspect paléographique, tout en étant le plus ancien témoin manuscrit connu en écriture arménienne, datable à peine plus d'un siècle après l'invention de l'alphabet arménien par saint Mesrop Maštoc' environ en l'an 405 après Jésus Christ.

Mais on peut aussi rappeler de véritables cas de bilinguisme et / ou de digraphisme. En documentant des phénomènes intéressants de coexistence entre des cultures différentes et en nous révélant parfois des scribes qui ont à l'évidence reçu une double éducation à la fois linguistique et graphique, en grec et dans une des autres langues de l'Orient chrétien, ils revêtent un intérêt particulier. Un premier cas révélateur en ce sens est celui du manuscrit d'Athènes, Gennadeios Bibliotheke, I.5, un codex des Évangiles de très petite taille, qui est d'un intérêt certain également du point de vue de l'histoire de la miniature : il figure, en effet, parmi les très rares manuscrits grecs datés qui soient attribuables à l'époque du Royaume latin de Constantinople, une période dans laquelle on assista à une crise profonde de la production de manuscrits grecs de luxe et dans laquelle on eut, de manière générale, un effondrement de la production de livres grecs. Or, ce manuscrit de la Gennadeios Bibliotheke a été transcrit à Césarée de Cappadoce en 1226 par le protonotaire Basile Mélitèniote, qui a signé le manuscrit non seulement en grec mais aussi en arménien ${ }^{17}$. Face à un témoignage de bilinguisme et de digraphisme gréco-arménien qui nous vient d'une région comme celle de Césarée de Cappadoce - l'actuelle Kayseri turque -, la tentation est naturellement très forte de voir même dans le protonotaire Basile Mélitèniote un arménien : c'est ce qu'a pensé Dimitri Korobeinikov, qui a exposé sa thèse dans un excellent article récent, en $2009^{18}$. On a, certes, affaire ici à un manuscrit qui documente la production chrétienne de livres grecs au XIII ${ }^{\mathrm{e}}$ siècle au sein du Sultanat de Rūm, dans une région de l'Anatolie qui était hors du contrôle byzantin, et dans un milieu où les phénomènes d'échange culturel avec le monde arménien sont très forts. Mais l'onomastique qui

17. F. D’Aiuto, A. Sirinian, «Un carme bizantino in onore degli evangelisti e la sua versione armena nel Vat. gr. 1445 », Rivista di studi bizantini e neoellenici, n.s. 36 (1999), p. 121-169 avec 12 pl. h.t., réimpr. dans Tra Oriente e Occidente. Scritture e libri greci, p. 297-347, avec 12 pl. h.t.

18. D. Korobeinikov, "A Greek Orthodox Armenian in the Seljukid Service: the Colophon of Basil of Melitina », dans R. Shukurov [Šukurov] (éd.), More i berega. K 60-letiju Sergeja Pavloviča Karpova ot kolleg i učenikov - Mare et litora. Essays Presented to Sergei Karpov for his 60th Birthday, Moscou, 2009, p. 709-724. 
est attestée dans le colophon - les noms d'Oreste et de Sophie, les parents du copiste Basile Mélitèniote - est substantiellement étrangère au monde arménien. Il est plus difficile alors de penser que nous avons ici affaire à une famille d'Arméniens chalcédoniens. Enfin, alors que le texte grec du manuscrit, y compris celui du colophon, est écrit par Basile dans une écriture très fine et extrêmement régulière, qui est un véritable chef-d'œuvre de micrographie avec des lettres qui ont un millimètre de haut, les deux souscriptions arméniennes sont écrites dans une écriture arménienne commune de type bolorgir et de module moyen (ou en majuscule erkat 'agir pour les quelques lignes initiales du colophon arménien principal) : l'écriture arménienne, en bref, n'est pas exécutée avec une élégance ou une habileté particulière. En conclusion, on serait tenté de dire que Basile Mélitèniote était plutôt un Grec et que, dans ce manuscrit, il aura simplement voulu fournir un témoignage des compétences qu'il avait acquises aussi dans le domaine de l'écriture arménienne, un autre champ linguistique auquel il devait évidemment avoir affaire dans sa position de protonotaire de la section chrétienne grecque de la chancellerie du Sultanat de Rūm, dans un milieu tel que celui de Césarée de Cappadoce, qui devait naturellement favoriser ce genre d'interactions linguistiques et graphiques.

Un autre exemple significatif qu'on peut rappeler ici est celui d'un manuscrit gréco-arménien de la Bibliothèque nationale de France qui est considéré comme autographe, pour l'arménien, d'un intellectuel et écrivain arménien de premier rang de la fin du XII ${ }^{\mathrm{e}}$ siècle, Nersēs Lampronac'i, le fils cadet d'Oshin II, seigneur de Lampron († 1170), qui devint, en 1176, évêque de la ville de Tarse en Cilicie, siège sur lequel il restera jusqu'à sa mort en juillet 1198. En effet, dans le Parisinus arménien 27 - un manuscrit du Nouveau Testament (Actes, Épîtres et Apocalypse) sur deux colonnes, dont l'une, à gauche, est occupée par le texte grec, l'autre, à droite, par sa version arménienne -, on lit le suivant colophon arménien :

$<$ Moi, $>$ Nersēs, humble évêque de Tarse, j'ai travaillé amoureusement pour ce livre sacré, que j'ai trouvé vieilli en grec, et que j'ai renouvelé en arménien. Que je puisse être estimé digne de la grâce des prières de vous qui en profiterez [ $=$ les lecteurs à venir].

Une nouvelle analyse paléographique du texte arménien du manuscrit - que nous avons comparé avec quelques autres autographes de Lampronac'i conservés à Erevan, à Venise et au Vatican - ne laisse pas de doutes sur l'identité du copiste arménien, qui est effectivement Nersēs lui-même, tandis que le texte grec du Nouveau Testament a été à l'évidence copié, comme l'analyse de l'écriture grecque suggère,

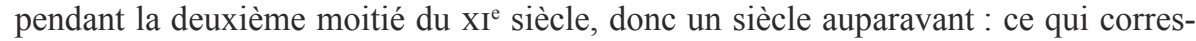
pond exactement à ce que, comme nous l'avons vu, Nersēs nous dit dans son colophon, à savoir qu'il avait trouvé le manuscrit « vieilli en grec » et qu'il l'a « renouvelé en arménien ». De plus, élément plus intéressant dans notre perspective, un examen détaillé du manuscrit parisien m'a permis d'identifier aussi dans ses pages une main grecque qui est probablement celle de Lampronac'i même : dans quelques passages, en effet, on dirait que Nersēs - ayant remarqué, grâce à l'arménien, qu'il y avait de petites lacunes dans le texte grec - a intégré de sa main dans la marge, pendant le processus de transcription du texte arménien, le texte grec manquant (c'est ce qu'on peut observer par exemple aux f. $173 \mathrm{v}$ et $179 \mathrm{v}$ ). 
Enfin, pour terminer ce bref exposé dédié aux Graeca dans les manuscrits orientaux, on peut rappeler - indépendamment du cas des manuscrits bilingues proprement dits - la présence qui est également assez fréquente de « traces » en grec comme, par exemple, des ajouts ou des annotations de nature différente, ou des signatures de cahiers, ou des parchemins réutilisés qui proviennent de manuscrits grecs et qui, dans ces manuscrits orientaux, sont remployés comme folios de garde ou bien comme feuillets palimpsestes : ce qui représente un témoignage significatif d'une vaste circulation de la littérature et de la production manuscrite grecques bien au delà des « frontières » linguistiques qu'on considérerait comme naturelles. On ne s'étonnera donc pas de constater qu'un recensement orienté vers la recherche de Graeca dans les fonds manuscrits orientaux de nos bibliothèques peut aussi conduire à des trouvailles philologiques de quelque importance, en nous restituant parfois des textes grecs nouveaux, comme ce fut le cas du Ménandre palimpseste qui fut découvert en 2003 dans un manuscrit syriaque de la Bibliothèque Vaticane (Vat. syr. 623) ${ }^{19}$.

19. F. D’Aiuto, « Graeca in codici orientali della Biblioteca Vaticana (con i resti di un manoscritto tardoantico delle commedie di Menandro) », dans Tra Oriente e Occidente. Scritture e libri greci, p. 227-296, avec 20 pl. h.t. 